\title{
Enfermedad diarreica aguda en pacientes con nutrición enteral en Unidad de Cuidados Intensivos: serie de casos
}

\section{Case Studies of Acute Diarrhea in ICU Patients on Enteral Nutrition}

Robin Germán Prieto 0., MD¹, Ángela María Navas C., MD², Fredy Orlando Mendivelso D., MD, MSc³

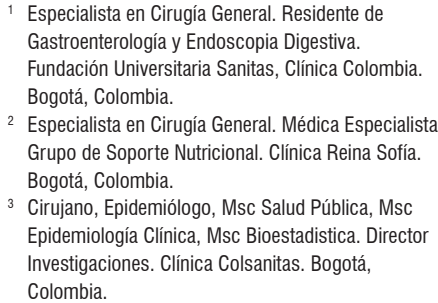

2 Especialista en Cirugía General. Médica Especialista Grupo de Soporte Nutricional. Clínica Reina Sofía. Bogotá, Colombia.

3 Cirujano, Epidemiólogo, Msc Salud Pública, Msc Epidemiología Clínica, Msc Bioestadistica. Director Investigaciones. Clínica Colsanitas. Bogotá Colombia.

\begin{abstract}
Resumen
La administración de nutrición enteral (NE) se menciona de modo frecuente como causa de aparición de diarrea en el paciente en la Unidad de Cuidados Intensivos (UCI), por lo cual existe la tendencia a disminuir su aporte 0 incluso suspenderla, conducta que en muchas ocasiones causa un retraso en la recuperación nutricional del paciente, incrementando además el riesgo de infecciones asociadas.

Realizamos un estudio descriptivo de serie de casos, realizado en la UCI de la Clínica Reina Sofía entre los meses de julio y octubre de 2015, siendo el principal criterio de inclusión la aparición de diarrea en pacientes a quienes se les inició NE en la UCl. El análisis estadístico se realizó mediante distribuciones de frecuencias absolutas y relativas expresadas en porcentajes. En variables cuantitativas se utilizaron medidas de tendencia central (promedio y mediana) y medidas de dispersión (rango y desviación estándar). La normalidad se analizó con el estadístico de Shapiro-Wilks. Hay que agregar que durante el período de estudio se identificaron siete pacientes con NE y episodios de EDA. La edad promedio de los pacientes incluidos fue de 73 años. Sin embargo, en ninguno de los casos se pudo establecer una relación directa entre los episodios de EDA y la administración de nutrición enteral.
\end{abstract}

\section{Palabras clave}

Nutrición enteral, enfermedad diarreica aguda, Unidad de Cuidados Intensivos.

\section{Abstract}

The administration of enteral nutrition (EN) is frequently mentioned as a cause of diarrhea in patients in Intensive Care Units (ICUs). Because of this, there is a tendency to reduce the use of EN or to even suspend it which often delays patients' nutritional recovery and incurs risks of associated infections.

This is a descriptive study of seven patients that was conducted in the ICU of the Clínica Reina Sofía from July to October 2015. The main criterion for inclusion was the occurrence of diarrhea in patients had been started on EN in the ICU. Statistical analyses used distributions of absolute and relative frequencies expressed in percentages. Measures of central tendency (mean and median) and measures of dispersion (range and standard deviation) were used for quantitative variables. The Shapiro-Wilk test was used to analyze normality.

During the study period seven patients receiving EN who had episodes of diarrhea were identified. The average age of the patients was 73 years. No direct relationships between episodes of of diarrhea and administration of enteral nutrition could be established.

\section{Keywords}

Enteral nutrition, acute diarrhea, Intensive Care Unit. 


\section{INTRODUCCIÓN}

La diarrea es una de las complicaciones gastrointestinales no hemorrágicas más frecuentes en los pacientes atendidos en una UCI, con una incidencia que de acuerdo con algunos estudios varía entre un 5\%-64\%. Las complicaciones de la diarrea pueden ser graves e incluyen infecciones en la piel, trastornos hidroelectrolíticos, deshidratación, desnutrición, e hipoalbuminemia. Todas estas alteraciones conducen a un aumento en el tiempo de hospitalización y mayores tasas de morbilidad y mortalidad.

Son múltiples las causas relacionadas con la diarrea en el paciente en Unidad de Cuidados Intensivos. La administración de nutrición enteral ha sido sobredimensionada como causa de la diarrea, por lo cual existe la tendencia a disminuir su aporte o incluso suspenderlo, acción que va a retrasar la recuperación nutricional del paciente, incrementando además el riesgo de infecciones asociadas, ya sea por alteraciones estructurales en la mucosa intestinal, como por el deterioro del estado nutricional del paciente.

Se han identificado otras causas de diarrea en relación con el uso de una gran variedad de medicamentos, especialmente los antibióticos, así como causas infecciosas, especialmente relacionadas con el Clostridium dificcile. Vale la pena mencionar que en nuestro medio desconocemos las características del paciente que presenta diarrea y que recibe nutrición enteral durante su atención en la UCI.

\section{MATERIALES Y MÉTODOS}

Estudio descriptivo de serie de casos. Se incluyeron pacientes adultos admitidos en UCI entre los meses de julio y octubre de 2015 en la Clínica Reina Sofía (Institución de alta complejidad en la ciudad de Bogotá D.C.). El principal criterio de inclusión fue el inicio de Nutrición Enteral (NE) en UCI. Se excluyeron pacientes con episodios de Enfermedad Diarreica Aguda (EDA) 48 horas previas al ingreso a UCI. El análisis estadístico se realizó mediante distribuciones de frecuencias absolutas y relativas expresadas en porcentajes. En variables cuantitativas se utilizaron medidas de tendencia central (promedio y mediana) y medidas de dispersión (rango y desviación estándar). La normalidad se analizó con el estadístico de Shapiro-Wilks.

\section{RESULTADOS}

Durante el período de estudio se identificaron siete pacientes con NE y episodios de EDA. La edad promedio de los pacientes incluidos fue 73 (D.E. \pm 13 ) años. La mayoría de los pacientes eran hombres $(71,4 \%)$ (tabla 1$)$. La estancia en UCI varió entre 13 y 36 días, y el tiempo de administración de la NE entre 11 y 32 días (figura 1). La EDA apare- ció entre el día 4 y 17 luego de iniciada la NE, y su duración osciló entre 2 y 20 días (figura 2). Como es de esperarse en paciente de UCI, todos estaban polimedicados en relación con la gravedad de sus patologías y comorbilidades.

Cuatro de los siete pacientes recibieron picosulfato de sodio días previos al inicio de la EDA; dos de ellos además recibieron enemas evacuadores, los dos medicamentos como manejo de ausencia de deposiciones por más de cuatro días. Otro paciente recibió lactulosa, previo al inicio de la EDA. En todos los pacientes se observó elevación de la proteína $\mathrm{C}$ reactiva y en la mayoría de ellos elevación de la procalcitonina. Solo en un paciente se sospechó infección por Clostridium dificcile pero la sospecha fue descartada mediante realización de coprocultivo y estudio de toxinas.

Ahora bien, en ninguno de los casos se pudo establecer una relación directa entre los episodios de EDA y la administración de NE. La mortalidad se presentó en dos de los cinco pacientes de género masculino (40\%) y en ninguna paciente femenina, para una mortalidad global de 28,5\%; en ningún caso la mortalidad se correlacionó con la EDA o con la administración de NE, y más bien se debió a la patología de base de los pacientes y sus complejas comorbilidades.

\section{DISCUSIÓN}

La palabra diarrea proviene del latín diarrhoea y es definida por la Real Academia Española de la Lengua como: "síntoma o fenómeno morboso que consiste en evacuaciones de vientre líquidas y frecuentes". La Organización Mundial de la Salud define la diarrea como: "la expulsión en 24 horas de tres (o más) deposiciones que adopten la forma del recipiente que las contiene; o que incluso se vuelven líquidas; y que pueden estar acompañadas (o no) de sangre". En la literatura médica pueden encontrarse más de 30 definiciones (1), lo cual evidencia la falta de un consenso mundial que facilite el estudio y las conclusiones con relación a esta alteración que se observa en los pacientes atendidos en cualquier Unidad de Cuidado Intensivo (UCI) y que en forma frecuente se correlaciona con la administración de Nutrición Enteral (NE) aunque no siempre sea esta la causa (2).

La nutrición enteral es la vía nutricional utilizada en el $46 \%$ al $77 \%$ de los pacientes de UCI, independientemente de su patología de base. Se ha demostrado que la misma, administrada de manera temprana, contribuye a la preservación de la mucosa intestinal y de la barrera inmunológica, disminuyendo el riesgo de sepsis y Falla Orgánica Múltiple (FOM), y favoreciendo los procesos de cicatrización $(3,4)$. Sin embargo, la NE ha sido asociada con la aparición de diarrea, la cual es una de las complicaciones gastrointestinales no hemorrágicas más frecuentes en los pacientes atendidos en una UCI, con una incidencia que varía entre un 5\%-64\% (5), y puede ser hasta del 95\% (6). Esta variación en los 
Tabla 1. Características clínicas de los pacientes con Nutrición Enteral y Enfermedad Diarreica Aguda en UCI.

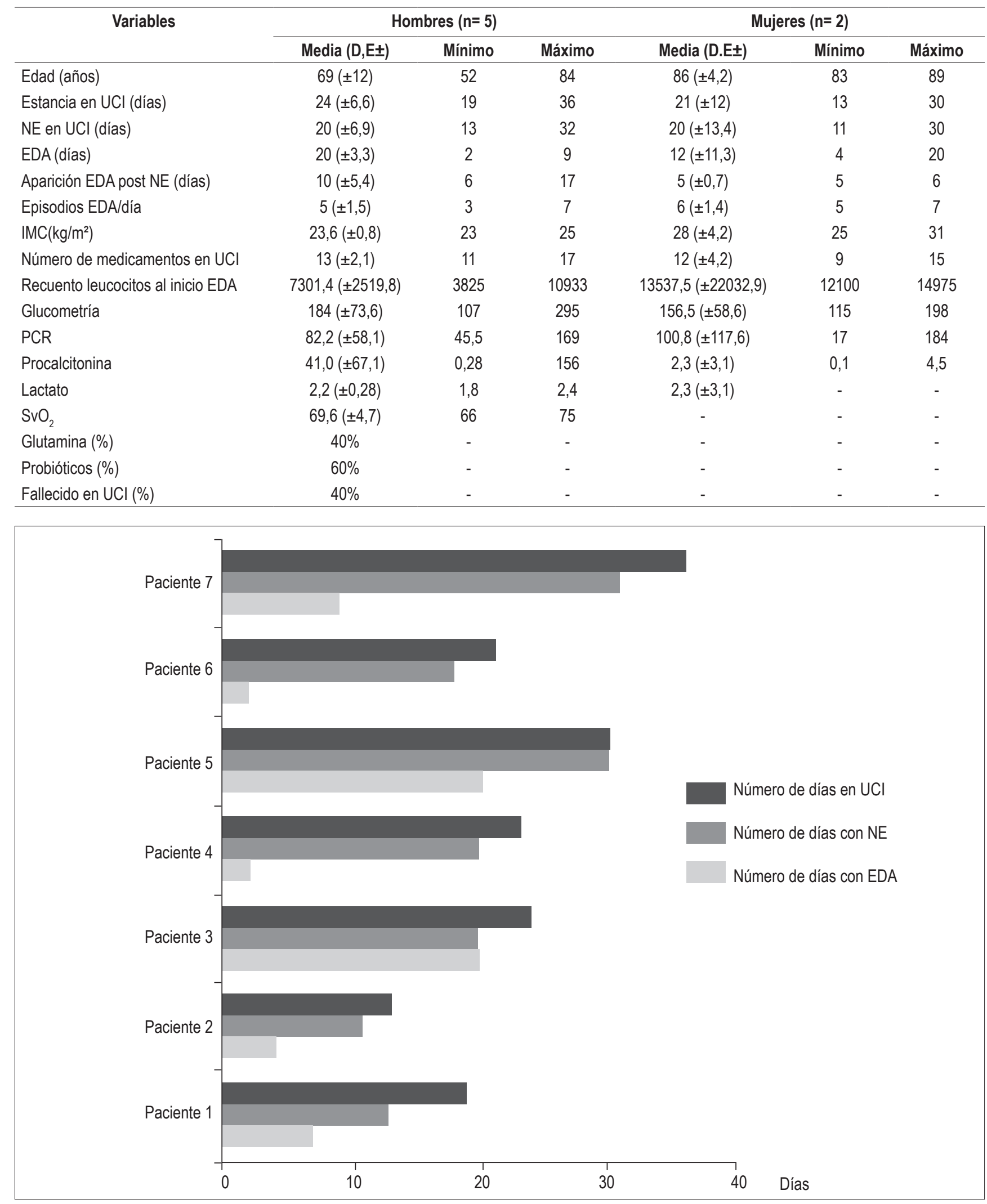

Figura 1. Descripción individual de la estancia en UCI, tiempo de manejo con NE y días con EDA. 


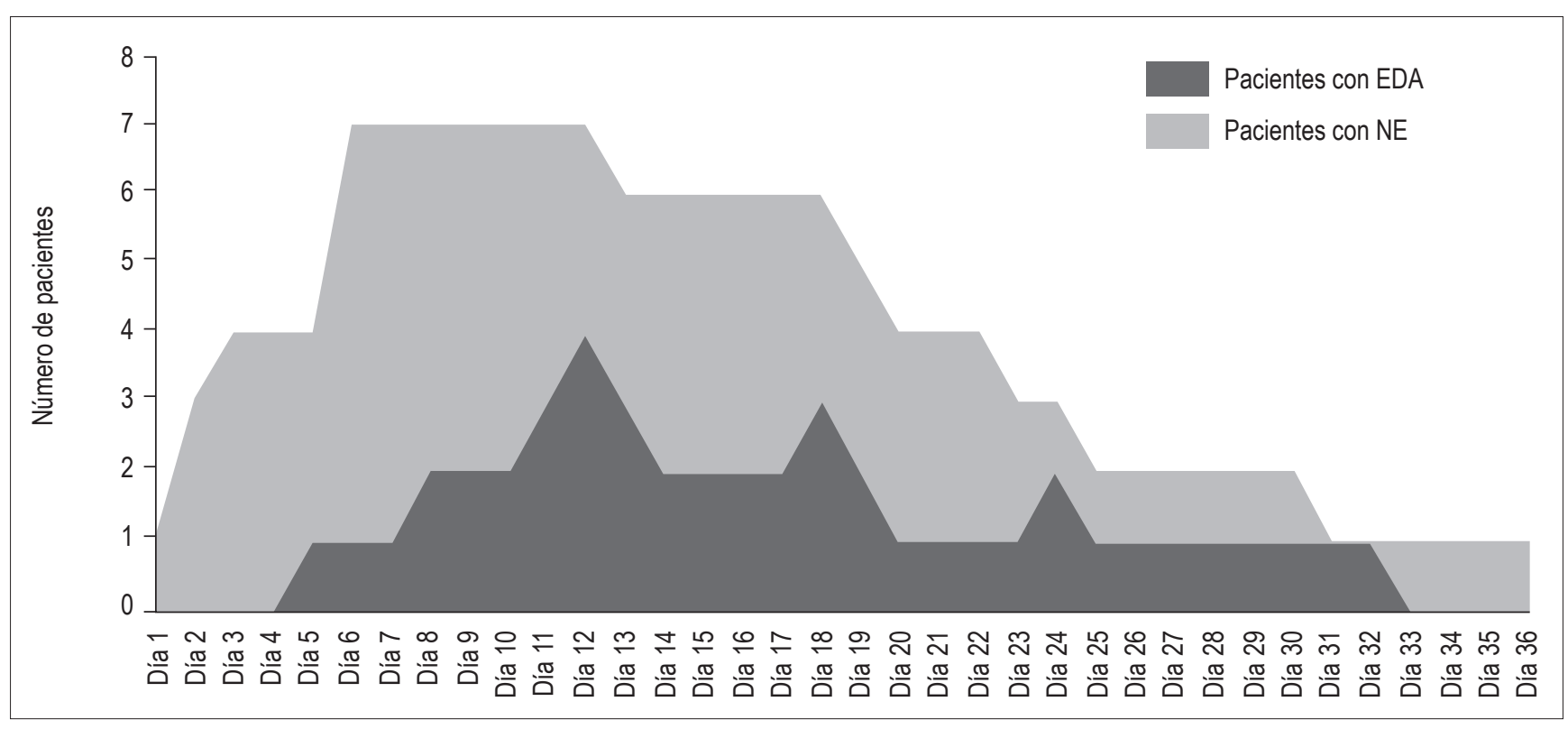

Figura 2. Distribución de frecuencia en el manejo con Nutrición Enteral y la aparición de la Enfermedad Diarreica Aguda en pacientes en UCI.

porcentajes se debe a la multitud de definiciones existentes de diarrea, que incluyen conceptos como frecuencia en las deposiciones (más de 3 o 4 veces al día), consistencia (semilíquida, líquida), peso (más de 300 gr por día), o la combinación de estas características. Se han diseñado múltiples escalas y de igual forma se han realizado estudios que clasifican las deposiciones, siendo quizás las más utilizadas, la escala de Bristol que las gradúa en una escala de 0 a 7 , donde los grados 6 y 7 corresponden a deposiciones diarreicas (7), y la escala del Colegio Real de Londres que las clasifica de acuerdo con su forma, peso y consistencia (8).

Son pocos los estudios que han evaluado la diarrea en los pacientes atendidos en la UCI o como complicación de la NE. Borges y colaboradores publicaron en 2008 un estudio observacional prospectivo, realizado en Brasil con 457 pacientes. Allí encontraron una incidencia de la diarrea asociada con la nutrición enteral del $29,5 \%$, (se definió la diarrea como la ocurrencia de más de dos evacuaciones diarias de aspecto líquido por un período mayor a 48 horas) (9). En 2011 Izaguirre y colaboradores realizaron un estudio de iguales características en 25 UCI de España, encontrando una incidencia del $6,4 \%$, (se definió diarrea como la presencia de 3 o más deposiciones líquidas en un período de 24 horas) (10).

\section{ETIOLOGÍA}

Desde el inicio de la nutrición enteral, las fórmulas nutricionales fueron consideradas como potenciales causantes de diarrea en los pacientes de UCI. Este concepto inicial- mente generalizado ha sido desvirtuado en la actualidad gracias a múltiples estudios que han evidenciado diversas causas de la diarrea (tabla 2) que ocurre en pacientes tratados en UCI y que reciben NE (11-13). Cabe anotar que muchos autores consideran que la principal causa de la diarrea se debe a los medicamentos que recibe el paciente crítico. Los antibióticos, especialmente los de amplio espectro, pueden disminuir la flora microbiana enteral, causando una alteración de los carbohidratos y aminoácidos en el metabolismo, por lo cual se disminuye la formación de ácidos grasos de cadena corta, importantes coadyuvantes en la absorción de agua y electrolitos por parte del intestino. Aunque en varios estudios se ha mencionado el uso de laxantes, proquinéticos y bloqueadores neuromusculares como causantes de diarrea, en un estudio retrospectivo realizado en Australia no se encontró dicha correlación (14). En un estudio prospectivo realizado en el mismo país, se analizaron 180 pacientes tratados en UCI, y se encontró una baja correlación entre el uso de metoclopramida o eritromicina usados como proquinéticos en forma individual, pero se observó una mayor casuística de diarrea cuando se usaban de forma conjunta (15). Muchos medicamentos de presentación líquida como el acetaminofén, el sulfato ferroso, la isoniazida, son hipertónicos, por contener jarabe o sorbitol. Algunas presentaciones en tabletas contienen lactosa y se constituyen en sustancias de alta carga osmolar con un efecto laxante osmótico.

Las infecciones bacterianas, parasitarias o micóticas, se constituyen en otra causa importante, aunque son pocos los estudios que han determinado de forma porcentual su 
Tabla 2. Principales causas de diarrea en el paciente que recibe nutrición enteral en la Unidad de Cuidado Intensivo.

\begin{tabular}{|c|c|c|c|c|}
\hline Medicamentos & Infecciones & Comorbilidad & $\begin{array}{l}\text { Relacionados con la } \\
\text { dieta }\end{array}$ & $\begin{array}{l}\text { Relacionados con la } \\
\text { técnica }\end{array}$ \\
\hline Antibióticos & Bacterianas & Isquemia intestinal & Hiperosmolaridad & Infusión "en bolos" \\
\hline Sales orales de Mg & Virales & Hipoperfusión intestinal & $\begin{array}{l}\text { Elevado contenido en } \\
\text { grasa }\end{array}$ & Infusión rápida transpilórica \\
\hline Suplementos de fosfato & Parasitarias & Obstrucción intestinal incompleta & Bajo contenido en sodio & $\begin{array}{l}\text { Infusión de dietas con baja } \\
\text { temperatura }\end{array}$ \\
\hline Antiácidos & Micóticas & Hipoalbuminemia & & \\
\hline Proquinéticos & Infección por C. difficile & Mala absorción & & \\
\hline Antiarrítmicos & Contaminación de la dieta & Alteraciones en la secreción biliar & & \\
\hline Inotrópicos & & $\begin{array}{l}\text { Alteraciones en la secreción } \\
\text { pancreática }\end{array}$ & & \\
\hline Antihipertensivos & & Ventilación mecánica & & \\
\hline Inmunosupresores & & Falla orgánica múltiple & & \\
\hline \multicolumn{5}{|l|}{ AINES } \\
\hline Agentes hiperosmolares & & & & \\
\hline
\end{tabular}

implicación (16). Uno de los microorganismos causales más mencionados es el Clostridium difficile, que en un estudio realizado en varias UCI de España solo representó el 5\% de las causas (10), también se ha encontrado infección por otros gérmenes como Salmonella, Shigella, Campylobacter y Escherichia Coli.

Las infecciones virales representan una causa importante de diarrea en el paciente en UCI, incluso en pacientes inmunocompetentes. Cuando ocurren de forma concomitante con una infección bacteriana se incrementa el riesgo de mortalidad. Los virus más implicados son el de la influenza, el sincitial respiratorio, el herpes simple, y el citomegalovirus (17)

En menor porcentaje otras alteraciones clínicas contribuyen a la aparición de diarrea: la isquemia intestinal relacionada con patología cardiovascular o con la arteriosclerosis mesentérica, común en la población adulta mayor; la hipoperfusión intestinal relacionada con el síndrome de bajo gasto cardíaco; la presencia de cualquiera de los síndromes de mala absorción intestinal; la falla orgánica múltiple; la ausencia de la válvula ileocecal por antecedente quirúrgico, así como el síndrome de intestino corto (18).

Muchos de los pacientes atendidos en UCI tienen algún grado de desnutrición. En estos pacientes es común encontrar alteraciones en la mucosa gastrointestinal que deterioran la digestión y la absorción de los nutrientes. Ocurren también alteraciones inmunológicas dadas por una disminución en los niveles de IgA que favorecen la proliferación microbiana y el paso de enterotoxinas al torrente sanguíneo a través de la barrera mucosa; todos estos factores pueden llevar a la aparición de diarrea (6).

\section{Nutrición enteral}

Por diversas razones la nutrición enteral ha sido implicada como causal de diarrea en el paciente crítico atendido en la UCI. Se han mencionado factores que dependen de la composición de la nutrición y que se relacionan con mayor riesgo de diarrea como son: la distribución de las calorías en los diferentes macronutrientes, la composición del aporte calórico dado por más del $20 \%$ de los carbohidratos y las grasas, la hiperosmolaridad dada por la presencia de solutos pequeños, bajos contenidos de vitamina $\mathrm{A}(<10000 \mathrm{UI} / \mathrm{L})$ o de sodio $(<90 \mathrm{mE} / \mathrm{L})$ (19). También se han implicado los altos contenidos de FODMAPs (fermentable oligo-, di-, and monosaccharides and polyols), como agentes laxantes (20).

Otros factores implicados son los relacionados con la técnica de administración: la administración por gravedad en la modalidad de bolos, y a altas dosis, incrementa el riesgo de diarrea; la utilización de bombas de infusión que regulan la administración de la nutrición puede disminuir este riesgo (5). Algunos autores relacionan la administración de la nutrición en yeyuno con un mayor riesgo de presentar diarrea (20), aunque en el estudio realizado por Montejo no se encontró variación porcentual si la administración de la nutrición se realizó en el estómago o en el yeyuno (12).

Por su composición, las fórmulas se convierten en un excelente caldo de cultivo, y ante la inadecuada manipulación de 
las fórmulas, o la no adherencia a los protocolos establecidos por cada UCI, especialmente en lo relacionado con el tiempo máximo de colgado de la mezcla, puede ocurrir colonización de la misma por múltiples gérmenes que van a ocasionar diarrea en el paciente crítico. Las fórmulas contaminadas, además de causar diarrea, pueden dar origen a infecciones respiratorias como la neumonía, una infección del tracto urinario, e incluso sepsis (14). Se ha estudiado una respuesta anormal de la absorción de agua en el colon, asociada con la nutrición enteral gástrica: la infusión de nutrición enteral al estómago aumenta la secreción de agua en el colon ascendente, situación que puede estar relacionada con la disminución de la secreción de péptido YY, un polipéptido intestinal que promueve la absorción colónica de agua. Durante la nutrición gástrica por sonda hay una disminución de la secreción de este péptido. La administración de ácidos grasos de cadena corta (producidos normalmente por las bacterias del colon) puede revertir esta situación, aumentando la absorción de agua en el colon (21).

\section{DIAGNÓSTICO}

La mayoría de las diarreas que ocurren en la UCI son autolimitadas en un período no mayor a 72 horas. El examen físico continúa siendo de gran importancia y en él se deben evaluar la presencia de deshidratación, de distensión abdominal o de signos de irritación peritoneal (22).

Una vez realizado el diagnóstico de diarrea en el paciente con NE en la UCI, se debe evaluar la etiología de acuerdo con las posibles causas presentadas en la tabla 1. Revisar qué tipo de medicamentos está recibiendo el paciente, poniendo especial atención en el uso de antibióticos y su tiempo de administración, ya que estos pueden estar relacionados con la presencia de colitis pseudomembranosa por Clostridium dificcile o por otros microorganismos. Se debe evaluar la administración de proquinéticos y de laxantes, así como de medicamentos hiperosmolares (22).

La presencia de procesos infecciosos intestinales se debe evaluar realizando el análisis de la materia fecal mediante estudios coproscópicos y coprocultivos, especialmente para Clostridium difficile. Se deben tomar muestras de sangre para evaluar la presencia de leucocitosis y para determinar alteraciones hidroelectrolíticas. En algunas ocasiones es de utilidad la realización de radiografías simples de abdomen para evaluar procesos obstructivos, así como la realización de colonoscopia para el estudio de las colitis (23).

La administración de NE deberá considerarse como causante de la diarrea, una vez descartadas las causas anteriormente mencionadas. Deben evaluarse las características de la mezcla en cuanto a su composición, al volumen administrado y al tipo de infusión sea continuo o en bolos, al igual que el tiempo de colgado de la nutrición para descartar contaminación de la mezcla (24).

\section{TRATAMIENTO}

Teniendo en cuenta que las diarreas pueden ser transitorias, el primer paso consiste en hacer un seguimiento sin una intervención terapéutica definida, aunque desde el inicio de la diarrea se debe realizar una adecuada reposición de agua y electrolitos y se deben analizar los medicamentos que recibe el paciente para plantear variaciones en las formas farmacéuticas o los compuestos activos de los mismos (25).

Quizás el tratamiento más específico se puede realizar en el caso de infección por Clostridium dificcile que, de acuerdo con su gravedad, se puede clasificar en leve (no requiere más que la suspensión del antibiótico), moderada (requiere tratamiento con metronidazol) y grave (cuando se requiere realizar el tratamiento con vancomicina) $(16,26)$.

\section{CONCLUSIONES}

Aunque la diarrea es una de las complicaciones más frecuentes en los pacientes atendidos en las UCI, a nivel mundial son relativamente pocos los estudios que se han realizado para evaluar las características de la diarrea en el paciente atendido en UCI que recibe nutrición enteral. La recomendación actual en el manejo de la NE en el paciente con diarrea, es mantenerla. Se puede variar la composición o la técnica de administración, pero no se recomienda la suspensión de la misma, ya que el reposo intestinal produce alteraciones en la integridad de la mucosa y favorece el sobrecrecimiento bacteriano, perpetuando el cuadro diarreico (27). Aunque algunos estudios sugieren el uso de probióticos y prebióticos para el tratamiento de la diarrea, la evidencia no es clara y faltan estudios adicionales al respecto. El uso de fibra soluble puede ser útil en algunos pacientes que reciben nutrición enteral $(28,29)$.

Como nuestra presentación es una revisión de casos, entendemos que la misma no nos permite sacar conclusiones estadísticamente significativas con relación al uso de la NET y la aparición de la diarrea pero resaltamos que en ninguno de nuestros pacientes se pudo establecer una relación directa entre los episodios de EDA y la administración de nutrición enteral. Asimismo, consideramos de gran importancia la realización de estudios que diseñen un protocolo para la evaluación y el manejo de la diarrea en UCI, el cual evitará suspensiones innecesarias de la nutrición enteral y permitirá establecer un diagnóstico $y$ un tratamiento oportuno de condiciones corregibles. Consideramos de gran utilidad la realización de estudios prospectivos que permitan evaluar de manera objetiva las 
características del paciente atendido en UCI que presenta diarrea, y su correlación con la administración de la nutrición enteral en nuestro medio hospitalario.

\section{REFERENCIAS}

1. Lebak KJ, Bliss DZ \& Savik K. What's new on defining diarrhea in tube-feeding studies? Clin Nurs Res. 2003;12(2),174-204.

2. Whelan K, Judd PA, Taylor MA. Defining and reporting diarrhoea during enteral tube feeding: do health care professionals agree? Journal of Human Nutrition and Dietetics. 2003;16(1):21-6.

3. Luft VC, Beghetto MG, de Mello EZ, Polanczyk CA. Role of enteral nutrition in the incidence of diarrhoea among hospitalized adult patients. Nutrition. 2008;24(6):528-35.

4. Martin B. Prevention of gastrointestinal complications in critically ill patients. AACN Advanced Critical Care. 2007;18(2):158-66.

5. Martinuzzi ALN, Ferraresi Zarranz EM, Santana Porbén S, Alcántara S, Alonso M. Diarrea en el paciente crítico. Su actualidad. RCAN Rev Cubana Aliment Nutr. 2012;22(1):120-134.

6. Whelan K, Judd PA, Tuohy KM, Gibson GR, Preedy VR, Taylor MA. Fecal microbiota in patients receiving enteral feeding are highly variable and may be altered in those who develop diarrhea. American Journal of Clinical Nutrition. 2009;89(1):240-7.

7. Heaton K. Bristol stool form scale. Family Doctor Books in association with the British Medical Association. Preview Books Online; 2003. Bowels [Online]. Available: http:// www.familydoctor.co.uk [Accessed 1st July 2003].

8. Whelan K, Judd PA, Taylor MA: Assessment of fecal output in patients receiving enteral tube feeding: validation of a novel chart. European Journal of Clinical Nutrition. 2004; 58(7):1030-1037.

9. Borges SL, Pinheiro BV, Pace FHL, Chebli JMF. Diarréia nosocomial em unidade de terapia intensiva: incidencia e fatores de risco. Arq Gastroenterol. 2008;45(2):117-24.

10. Izaguirre Guerricagoitia L, Truchuelo Aragón A. Prevalencia de diarrea en las unidades de pacientes en estado crítico de España: estudio multicéntrico. Enferm Intensiva. 2011; 22(2):65-73.

11. Ukleja A. Altered GI motility in critically ill patients: current understanding of pathophysiology, clinical impact, and diagnostic approach. Nutrition in Clinical Practice 2010;25(1):16-25.

12. Montejo JC. Enteral nutrition-related gastrointestinal complications in critically ill patients: a multicenter study. The Nutritional and Metabolic Working Group of the Spanish Society of Intensive Care Medicine and Coronary Units. Crit Care Med. 1999;27(8):1447.

13. Luft VC, Beghetto MG, de Mello ED, Polanczyk CA. Role of enteral nutrition in the incidence of diarrhea among hospitalized adult patients. Nutrition. 2008; 24(6):528-35.

14. Jack L, Coyer F, Courtney M, Venkatesh B. Diarrhoea risk factors in enterally tube fed critically ill patients: A retros- pective audit. Critical Intensive and Critical Care Nursing. 2010;26(6),327-334.

15. Nguyen N, Ching K, Fraser R, Chapman M, Holloway R. Risk of Clostridium difficile diarrhoea in critically ill patients treated with erythromycin-based prokinetic therapy for feed intolerance. Intensive Care Med. 2008; 34(1):169-173.

16. Cohen SH, Gerding DN, Johnson S,Kelly CP, Loo VG, Clifford McDonald MDL, Pépin J, Wilcox MH. Clinical practice guidelines for Clostridium difficile infection in adults: 2010 update by the Society for Healthcare Epidemiology of America (SHEA) and the Infectious Diseases Society of America (IDSA). Infect Control Hospital Epidemiol. 2010;31(5):431-55.

17. Miggins M, Hasan A, Hohmann S. The potential influence of common viral infections diagnosed during hospitalization among critically ill patients in the United States. PLoS One $2011 ; 29 ; 6(4)$ :e18890.

18. Montejo JC, Jiménez J, Ordóñez j, Caparrós T, García A, Ortiz C, López J; para el Grupo de Trabajo de Metabolismo y Nutrición de la SEMICYUC Sociedad Española de Medicina Intensiva, crítica y Unidades Coronarias. Complicaciones gastrointestinales de la nutrición enteral en el paciente crítico. Medicina Intensiva [España]. 2001;25:152-60.

19. Barrett JS, Shepherd SJ, Gibson PR. Strategies to manage gastrointestinal symptoms complicating enteral feeding. JPEN J Parenter Enteral Nutr. 2009;33(1):21-6.

20. Halmos EP, Muir JG, Barrett JS, et al. Diarrhoea during enteral nutrition is predicted by the poorly absorbed short-chain carbohydrate (FODMAP) content of the formula. Aliment Pharmacol Ther 2010;32(7):925-933.

21. Whelan K. Enteral tube feeding diarrhoea: manipulating the colonic microbiota with probiotics and prebiotics. Proceedings of the Nutrition society. 2007;66(3):299-306.

22. Kenneally C, Rosini JM, Skrupky LP, et al. An analysis of thirty-day mortality for Clostridium difficile-associated disease in the ICU setting. Chest. 2007;132(2):418-424.

23. McClave SA, Martindale RG, Vanek VW, et al. Guidelines for the Provision and Assessment of Nutrition Support Therapy in the Adult Critically Ill Patient: Society of Critical Care Medicine (SCCM) and American Society for Parenteral and Enteral Nutrition (A.S.P.E.N.). JPEN J Parenter Enteral Nutr 2009;33:277.

24. Rushdi TA, Pichard C, Khater YH. Control of diarrhea by fiberenriched diet in ICU patients on enteral nutrition: a prospective randomized controlled trial. Clin Nutr 2004; 23(6):1344-52.

25. O'Keefe SJ. A guide to enteral access procedures and enteral nutrition. Nat Rev Gastroenterol Hepatol. 2009;6(4):207-215.

26. Maroo S, Lamont JT. Recurrent clostridium difficile. Gastroenterology. 2006;130:1311-1316.

27. Whelana K, Schneiderb S. Mechanisms, prevention, and management of diarrhea in enteral nutrition. Current Opinion in Gastroenterology. 2011,27(2):152-159.

28. Barraud D, Blard C, Hein F, et al. Probiotics in the critically ill patient: a double blind, randomized, placebo-controlled trial. Intensive Care Med 2010;36(9):1540-1547.

29. Rushdi TA, Pichard C, Khater YH. Control of diarrhea by fiberenriched diet in ICU patients on enteral nutrition: a prospective randomized controlled trial. Clin Nutr 2004; 23(6):1344-52. 